$\stackrel{N}{N}$

Global Journals Inc.

कै

\title{
Characterization of Domestic Cats Patients Treated at the Veterinary Hospital of the Faculty of Veterinary Medicine and Zootechnics at the San Carlos of Guatemala University in 2019
}

By Pérez-Archila Karla María, Villatoro-Chacón Daniela Mariel \& Arizandieta-Altán Carmen Grizelda

Universidad de San Carlos de Guatemala

Abstract- The demand for pets to fill affective family spaces, the increased economic capacity of social classes and the displacement of rural populations to cities are influencing factors in increasing pet acquisition. This cohabitation can trigger epidemiological factors that affect human health. For this reason, it is necessary to know the diseases that affect domestic cats that live with humans and determine if there are zoonotic infectious agents that inflict on human health. In the present study, 103 medical records of domestic cats attended in 2019 at the Veterinary Hospital of the Faculty of Veterinary Medicine and Animal Zootechnics were studied. This in order to categorize the most frequent diseases in the species. $71.84 \%$ of the cats examined were sick. $55.41 \%$ were males, while the age range with the highest frequency was $0-1$ years. The most frequent category according to the VITAMIN D System was Inflamatory/infectious.

Keywords: diseases, domestic cats, VITAMIND.

GJMR-G Classification: NLMC Code: WC 900

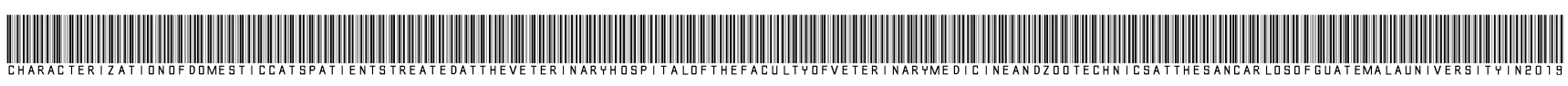

Strictly as per the compliance and regulations of:

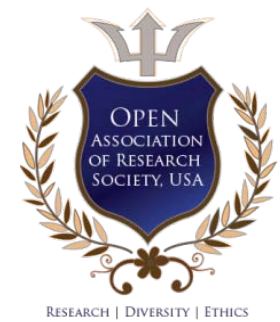

(c) 2020. Pérez-Archila Karla María, Villatoro-Chacón Daniela Mariel \& Arizandieta-Altán Carmen Grizelda. This is a research/review paper, distributed under the terms of the Creative Commons Attribution-Noncommercial 3.0 Unported License $\mathrm{http} / / / c r e a t i v e c o m m o n s . o r g / l i c e n s e s / b y-n c / 3.0 /$ ), permitting all non-commercial use, distribution, and reproduction in any medium, provided the original work is properly cited. 


\title{
Characterization of Domestic Cats Patients Treated at the Veterinary Hospital of the Faculty of Veterinary Medicine and Zootechnics at the San Carlos of Guatemala University in 2019
}

\author{
Caracterización de Gatos Domésticos Atendidos en el Hospital Veterinario de la \\ Facultad de Medicina Veterinaria y Zootecnia de la Universidad de San Carlos de \\ Guatemala en el año 2019
}

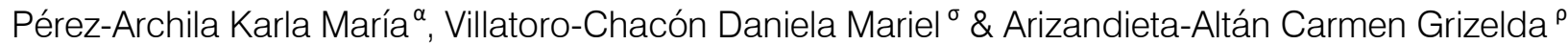

\begin{abstract}
Resumen- La demanda de mascotas para llenar espacios afectivos familiares, el aumento de la capacidad económica de las clases sociales y el desplazamiento de poblaciones rurales a las ciudades son factores influyentes en el incremento de la adquisición de mascotas. Esta cohabitación puede desencadenar factores epidemiológicos que afectan a la salud humana. Por esta razón, es necesario conocer las enfermedades que afectan a los gatos domésticos que conviven con el humano y determinar si existen agentes infecciosos zoonóticos que infieran en la salud humana. En el presente estudio se estudiaron 103 registros médicos de gatos domésticos atendidos en el año 2019 en el Hospital Veterinario de la Facultad de Medicina Veterinaria y Zootecnia. Esto con el fin de categorizar las enfermedades más frecuentes en la especie. El $71.84 \%$ de los gatos examinados estaban enfermos. El 55. 41\% fueron machos, mientras que el rango etario con mayor frecuencia fue de $0-1$ años. La categoría según el sistema VITAMIND con mayor frecuencia fue la inflamatoria/infecciosa (45.95\%). Los datos obtenidos sugieren que esta especie debe ser estudiada dado que las enfermedades de tipo infeccioso pueden ser un eslabón epidemiológico para la población humana.
\end{abstract}

Palabras clave: enfermedades, felinos domésticos, VITAMIND.

Abstract- The demand for pets to fill affective family spaces, the increased economic capacity of social classes and the displacement of rural populations to cities are influencing factors in increasing pet acquisition. This cohabitation can trigger epidemiological factors that affect human health. For this reason, it is necessary to know the diseases that affect domestic cats that live with humans and determine if there are zoonotic infectious agents that inflict on human health. In the present study, 103 medical records of domestic cats attended in 2019 at the Veterinary Hospital of the Faculty of Veterinary Medicine and Animal Zootechnics were studied. This in order to categorize the most frequent diseases in the species. $71.84 \%$ of the cats examined were sick. $55.41 \%$ were males, while the age range with the highest frequency was $0-1$ years. The most frequent category according to the VITAMIN D

Author a $\sigma$ p: Departamento de Ayudas Diagnósticas, Hospital Veterinario, Facultad de Medicina Veterinaria y Zootecnia, Universidad de San Carlos de Guatemala, ciudad. e-mail: danavilla47@gmail.com
System was Inflamatory/infectious. The data obtained suggests that this species should be studied since infectious diseases can be an epidemiological link for the human population.

Keywords: diseases, domestic cats, VITAMIND.

\section{INTRODUCCIÓN}

os felinos domésticos están dentro de los animales de compañía más comunes alrededor del mundo (1). En el 2006 la Asociación Americana de Medicina Veterinaria en Estados Unidos indicó que 6 de cada 10 familias poseían una mascota siendo el 54\% perros, gatos o ambos (2). En Guatemala se estimó hace más de 30 años una población de 32,758 felinos, dónde el índice habitantegato fue de 20.98 a 1, sólo en la Ciudad de Guatemala (3). Sin embargo, se debe considerar que ambas poblaciones han aumentado con los años al igual que el valor adquisitivo y sentimental hacia las mascotas.

Desde el punto de vista sanitario los gatos son susceptibles a padecer enfermedades de todo tipo (4). Además, tanto el perro como el gato son causantes de provocar enfermedades de tipo zoonótico. Se estima que de 1,400 patógenos que afectan a humanos, alrededor del 58\% son de origen zoonótico (5). La relación humano - animal hace susceptible a ambas especies a presentar enfermedades de origen infeccioso (6). Por esta razón, es importante conocer el comportamiento de las enfermedades en los gatos domésticos, para conocer el comportamiento epidemiológico de las mismas y tomar medidas preventivas.

En el presente estudio se evaluaron los registros clínicos de los gatos domésticos atendidos en el Hospital Veterinario de la Facultad de Medicina Veterinaria y Zootecnia de la Universidad de San Carlos de Guatemala en el año 2,019. Esto con el fin de categorizar las enfermedades que afectan a esta 
especie utilizando la regla nemotécnica VITAMIN D. Los resultados obtenidos son la primera fuente de información para investigaciones futuras respecto a la clínica diaria y el efecto epidemiológico en cuanto a la salud humana y animal.

\section{il. Materiales y Métodos}

El estudio se realizó en el Departamento de Ayudas Diagnósticas, Hospital Veterinario, Facultad de Medicina Veterinaria y Zootecnia, de la Universidad de San Carlos de Guatemala, ubicado en la zona 12 de la Ciudad de Guatemala, cuyas coordenadas son: latitud 14.584154 y longitud -90.562422 .

Se analizaron 103 expedientes de gatos domésticos atendidos en el Hospital Veterinario, durante el año 2019.

Se tomaron registros sobre las variables siguientes: sexo, edad, raza y se categorizaron las patologías que presentaba cada paciente de acuerdo a la regla nemotécnica VITAMIN D mediante su acrónimo: $\mathrm{V}=$ vascular; I= inflamatorio / infeccioso; $\mathrm{T}=$ traumático / tóxico; $A=$ anómalo / alérgico / autoinmune; $\mathrm{M}=$ metabólico; I = idiopático; $\mathrm{N}$ = neoplásico / nutricional; $\mathrm{D}=$ degenerativo (7).

Los pacientes con patologías diagnosticadas fueron agrupados según su edad de acuerdo al rango etario siguiente: 0 a 1 año, 1 a 5 años, 5 a 10 años, 10 a 17 años y sin especificar.
Para el diagnóstico de las enfermedades en los pacientes felinos, se tomaron en cuenta exámenes de laboratorio como: hematología, bioquímica sanguínea, examen de orina, imágenes diagnósticas y en los casos dónde no hubo ningún método diagnóstico se tomó como: sin diagnóstico.

Para el análisis de datos se utilizó estadística descriptiva utilizando distribuciones de frecuencias para las variables. Se utilizó el software estadístico SPSS versión 25.0.

\section{Resultados}

De los 103 pacientes atendidos durante el año 2019 , un $71.84 \%$ (74/103) fueron diagnosticados con alguna enfermedad, un $22.33 \% \quad(23 / 103)$ fueron clasificados como casos inconclusos por falta de pruebas para determinar el diagnóstico, un 5.83\% (6/103) fueron pacientes sanos.

En cuanto al sexo, el 46.6\% (48/103) de los gatos fueron hembras, el 52.43\% (54/103) fueron machos y el $0.97 \%$ (1/103) no se especificó en el registro clínico.

En cuanto a la raza, todos los pacientes evaluados (100\%) fueron sin raza definida (SRD). El promedio de edad de los pacientes fue de $3.65 \pm 0.4$. Sin embargo, se categorizó a los pacientes por rangos etarios (cuadro 1), siendo los pacientes entre 0 a 1 año con $44.6 \%$ con mayor prevalencia.

Cuadro 1: Rangos etarios de gatos domésticos atendidos en el Hospital Veterinario

\begin{tabular}{|c|c|c|}
\hline Rango etario & $\mathbf{N}$ & $\%$ \\
\hline 0 a 1 año & 33 & 44,59 \\
\hline 1 a 5 años & 21 & 28,38 \\
\hline 5 a 10 años & 10 & 13,51 \\
\hline 10 a 17 años & 8 & 10,81 \\
\hline Sin especificar & 2 & 2,70 \\
\hline
\end{tabular}

Según la regla nemotécnica VITAMIN D se encontró que el 45.95\% (34/74) fueron diagnosticados con patologías de tipo inflamatorio/infeccioso, siendo la mayor casuística del estudio, seguida de las patologías de tipo traumático/tóxico con un 31.08\% (23/74) y de tipo neoplásico/nutricional con un 13.51\% (10/74). En el cuadro 2 se describe la clasificación según VITAMIN D de los pacientes con diagnóstico clínico.

Cuadro 2: Clasificación de acuerdo a VITAMIND en gatos domésticos

\begin{tabular}{|c|c|c|}
\hline VITAMIND & N & $\%$ \\
\hline Vascular & 1 & 1,35 \\
\hline Inflamatorio/ Infeccioso & 34 & 45,95 \\
\hline Traumatico/ Tóxico & 23 & 31,08 \\
\hline Anómalo/Alérgico/Autoinmune & 2 & 2,70 \\
\hline Metabólico & 2 & 2,70 \\
\hline Idiopático & 1 & 1,35 \\
\hline Neoplásico/ Nutricional & 10 & 13,51 \\
\hline Degenerativo & 1 & 1,35 \\
\hline
\end{tabular}


La enfermedad vascular los casos observados fueron únicamente efusión pleural.

Dentro de las enfermedades de tipo inflamatorio/infeccioso las patologías observadas fueron las siguientes: criptosporidiosis, giardiasis, coccidiosis, enteritis bacteriana, ectoparásitos (pulgas), gastritis, leucemia viral felina, sida felino, inflamación de miembro torácico anterior, cistitis idiopática, infección urinaria, dermatomicosis, lesión infectada por mordida, neumonía, pancreatitis y discoespondilitis.

Las enfermedades de tipo traumático/tóxico diagnosticadas fueron principalmente fracturas, hematomas en piel, contusión medular, hernia diafragmática, heridas post-quirúrgicas, subluxaciones y compresión medular.

Para las enfermedades de tipo anómalo /alérgico /autoinmune se presentó dermatitis alérgica por picadura de pulga.

Respecto a las enfermedades de tipo metabólico se encontró un caso de encefalopatía hepática e hipertiroidismo. La única enfermedad diagnosticada para el tipo idiopático fue megacolon.

En cuanto a las enfermedades neoplásicas/ nutricionales se encontraron diagnósticos con síndrome paraneoplásico, neoplasias de tipo metastásicas pulmonares,urolitiasis asociada a dieta, neoplasias mamarias y linfoma.

Para el caso de las enfermedades de tipo degenerativo se observó un caso decardiomiopatía hipertrófica.

\section{Discusión}

Los datos generados sugieren que las enfermedades más frecuentes en los gatos domésticos atendidos en el Hospital Veterinario son de origen inflamatorio/infeccioso, seguido de las traumáticas/tóxicas y neoplásicas/nutricionales. Estos datos son similares a los observados en perros por Alvarado-Pérez et al. (8) en la ciudad de Guatemala. Esto puede indicar que ambas especies pueden tener un factor epidemiológico o ambiental en común (9).

La efusión pleural fue la única patología observada en los trastornos vasculares. Se utilizó la radiografía como método diagnóstico. Algunos autores han encontrado que la efusión pleural afecta más a gatos que perros. Esto puede deberse a que las causas más frecuentes son el linfoma, peritonitis infecciosa felina, piotórax, quilotórax, mesotelioma, hemotórax y fallo cardíaco congestivo derecho (10).

Las enfermedades infecciosas/inflamatorias fueron las más frecuentes siendo las endo y ecto parasitosis las más reportadas. Esta prevalencia concuerda con estudios donde se observó que en el $61.46 \%$ de gatos evaluados presentaban algún tipo de parásito gastrointestinal, siendo parásitos potencialmente zoonóticos (11). Las enfermedades virales como leucemia y el síndrome de inmunodeficiencia felina fueron la segunda patología observada en los pacientes en el estudio. Otros autores han reportado en el caso de leucemia viral felina una prevalencia entre el 1 al 8\%; mientras que el virus de inmunodeficiencia felina presenta prevalencias hasta del $14 \%$ en los gatos sin signología clínica (12). Otra enfermedad inflamatoria observada fue la cistitis idiopática, la cual es considerada la principal enfermedad de tracto urinario bajo en gatos. Algunos autores han reportado prevalencias del 1.77\% (13). La disco espondilitis también se observó en los pacientes del presente estudio. Esta enfermedad es poco diagnosticada en ésta especie y se han documentado solo 5 casos. Dentro de las causas de disco espondilitis más comunes están las infecciones de origen bacterianas siendo el $15 \%$ de la casuística total de disco espondilitis en gatos $(14,15)$. Existen diferentes formas de diagnóstico de esta patología, en el caso de éste paciente se utilizó radiografía simple en donde se observaron hallazgos radiológicos compatibles con discoespondilitis.

Para las patologías traumáticas/tóxicas, encontramos una prevalencia alta de fracturas de todo tipo, siendo las fracturas de huesos largos las más frecuentes. Los traumatismos por accidentes fueron el factor en común en estos pacientes. Además, se observó que algunos de ellos presentaban otras lesiones internas como neumotórax y contusión pulmonar (16). Las mielopatías evaluadas en la casuística del estudio fueron producidas principalmente por accidentes de auto además de las contusiones medulares y compresiones medulares. Pellegrino (2018), determinó que el trauma medular agudo producido por accidentes de autos es el trauma medular más frecuente en el gato.

La dermatitis alérgica a la pulga (DAPP), fue el único diagnóstico de la categoría alérgico/anómalo/autoinmune. La DAPP en gatos es la enfermedad alérgica más común en esta especie, manifestándose con alopecia inducida por el prurito (17), lo cual fue observado en los pacientes diagnosticados en el estudio.

La encefalopatía hepática fue uno de los trastornos metabólicos diagnosticados en el estudio. Este trastorno se presenta por alguna anomalía hepática en pequeños animales, causante de principalmente signos nerviosos, siendo una complicación neurológica, implica la translocación de toxinas del intestino al cerebro, es una patología de importancia clínica porque presenta entre sus signos, las convulsiones, que son un tema común de evaluación en la clínica diaria $(18,19)$. El hipertiroidismo se presentó en un paciente del estudio, publicaciones recientes, reportan que es la enfermedad endocrina más común en gatos, según Osorio y Matheus en 2012, 
determinaron que en países en vías de desarrollo, en el cual se encuentra Guatemala, la enfermedad no está muy diagnosticada, pero con el paso de los años, la población con gatos como mascotas aumenta, por lo que es importante tener presente la enfermedad; afecta a poblaciones de gatos adultos o geriátricos, presenta pérdida de peso, anorexia, polidipsia, polifagia, vómitos y diarrea $(20,21)$.

El megacolon idiopático fue el único diagnóstico de trastorno idiopático en los felinos del estudio. Esta patología tiene una incidencia del $60 \%$ a $70 \%$, es más frecuente en el gato que en el perro, puede ser primario o secundario, así como idiopático, donde no se encuentra lesión orgánica a la cual asociarla; produce como signología tenesmo, dificultad para defecar, depresión, anorexia, heces con sangre y moco, se presenta en edades variables, siendo la patología más frecuente en el gato relacionado a trastorno idiopático, es importante tomarla en cuenta como diferencial de patologías digestivas $(22,23)$.

Las neoplasias representan un porcentaje alto de motivo de consulta en la clínica diaria. Las neoplasias más frecuentes fueron metástasis pulmonar y neoplasias de glándula mamaria. La ocurrencia de tumores en gatos es de 158 a 470 de cada 100,000 animales y de éstos, cerca del $45 \%$ son tumores de piel y tejidos blandos. Para el caso de tumores mamarios, estos son los terceros más comunes después de los tumores hematopoyéticos y de piel (24). La urolitiasis en gatos, también fue parte del resultado del estudio. La causa más común de urolitos en gatos es la dieta que a su vez predispone a cálculos de estruvita. Se han observado prevalencias de hasta el 22\% de urolitiasis en gatos de diferentes minerales, siendo los más comunes estruvita y fosfato de calcio $(25,26)$; El linfoma felino se presentó en un paciente, los signos presentados varían, dependiendo del lugar de ubicación de la neoplasia, los más comunes son de presentación digestiva, donde se observa vómitos, diarrea, y pérdida de peso, por lo que es importante tomarlo en cuenta al momento de realizar un diagnóstico diferencial. Existen los linfomas de tipo mediastínico, multicentríco y extranodal $(27,28)$.

Para el caso de las enfermedades degenerativas, se observó un paciente con cardiomiopatía hipertrófica, caracterizada por la hipertrofia del corazón. Esta patología es la enfermedad más común diagnosticada por veterinarios, en muchos de los casos no se encuentra causa subyacente, por lo tanto, la mayoría son idiopáticos $(29,30)$.

\section{References Références Referencias}

1. Sparkes H, Cope K, Ellis S, et al. ISFM Guidelineson Population Management and Wel fare of Unowned Domestic Cats (Feliscatus). Journal of Feline Med. 2013. 15, 811-817. Reino Unido.
2. Voith V. The Impact of Companion Animal Problemson Society and the Role of Veterinarians. Vet Clinic Small. 2009. 39 327-345.

3. Galvez J. Características de la población canina y felina de la ciudad de Guatemala. Guatemala. Universidad de San Carlos de Guatemala; 1981.

4. Santillan M, Cruz I, Marin J, Lecumberri J. Frecuencia de géneros y especies de coccidias en heces de gatos en México, D. F. Vet. Mex. 1997. 28 (1).

5. Sarmiento L, Delgado L, Ruiz J, Sarmiento M, Becerra J. Parásitos intestinales en perros y gatos con dueño de la ciudad de Barranquilla, Colombia. RevInv Vet. 2008. 29 (4) 1403-1410.

6. Echeverry D, Giraldo M, Castaño J. Prevalencia de helmintos intestinales en gatos domésticos del departamento del Quindío, Colombia. 2012. 32: 430-6.

7. Pellegrino $F$, Pacheco E, Vazzoler E. Caracterización de los trastornos neurológicos en los perros: 1652 casos (Marzo 2008 - junio 210) Parte I. Revista Argentina NeuroVet. 2. 78-96. Argentina.

8. Alvarado-Pérez AM, Villatoro-Chacón DM, ChávezLópez JJ, Arizandieta-Altán CG. Caracterización de la población canina atendida en el centro municipal de atención canina de la ciudad de Guatemala. Rev. Electrón. vet. 2017; 18(12): 1-9. Recuperado de http://www.veterinaria.org/revistas/redvet/ n121217/121720.pdf

9. Backer LC, Grindem CB, Corbertt WT, Cullins L, Hunter JL. Petdogs as sentinels for envirommental contamination. Sci Total Environ. 2001; 274:161169.

10. Sanz L, Valenzuela C. Caracterización de los pacientes felinos con efusión pleural entre los años 2006 al 2010. Rev Hospitales Vet. 2011. 3 no. 2. Chile.

11. Diaz F, Ancassi M. Enteroparásitos en perros (Canisfamiliaris) y gato (Feliscatus) de la provincia de Puno. Journal of High. 2013.

12. Ávila N, Parra O, Barrios L, Bello M, Zambrano M, González A. Prevalencia de Leucemia Viral Felina, Inmunodeficiencia Viral Felina y Dirofilariasis en gatos refugiados en un albergue de animales en Maracaibo, Venezuela. Revista Científica [Internet]. 2015; XXV(4): 285-292. Recuperado de: https://w ww.redalyc. org/articulo.oa?id $=95941173002$

13. Kim Y, Kim H, Pfeiffer D, Brodbelt D. Epidemiologicalstudy of felineidiopathic cystitis in Seoul, South Korea. J Feline Med Surg. [Internet] 2018; 20(10): 913-921. Recuperado de https://pubm ed.ncbi.nlm.nih.gov/28967795/

14. Pellegrino F. Mielopatías en gatos. NEUROVET. [Internet] 2018. Recuperado de https://www.researc hgate.net/publication/325019645_Mielopatias_en_g atos 
15. Malik R, Latter M, Love D. Bacterial discospondylitis in a cat. Journal of Small Animal Prac. 31. 404 - 406. [Internet] 2008. Recuperado de https://www .researchgate.net/publication/229695399_Bacterial_ discospondylitis_in_a_cat/citation/download

16. Scott H. Repair of longbone fractures in cats. In Practice. [Internet] 2005; 27:390-397. Recuperado de: https://inpractice.bmj.com/content/27/8/390.info

17. Colombini S, Hodgin E, Foil C, Hosgood G, Foil L. Induction of felineflea allergy dermatitis and the incidence and histopathological characteristics of concurrentin dolentlipulcers. Vet Dermatol. [Internet] 2001; 12(3): 155-161. Recuperado de: https://pubmed.ncbi.nlm.nih.gov/11420931/

18. Gow A. Hepatic Encephalopathy. Vet Clin North Am Small Anim Pract. [Internet] 2017; 47(3):585-599. Recuperado de: https://pubmed.ncbi.nlm.nih.g ov/28069286/

19. Pellegrino F. Encefalopatía hepática en perros y gatos. AN. VET. (MURCIA) 25: 21-45. 2009. Recuperado de: https://revistas.um.es/analesv et/article/view/100161/95631

20. Osorio $H$, Matheus S. Actualización en el funcionamiento de la glándula tiroides en el gato domestico, segunda parte: hipertiroidismo felino. Biosalud. Vol 11 No. 1, 2012. pags. 57-70. recuperado de http://vip.ucaldas.edu.co/biosalud/d ownloads/Biosalud_11(1)_completa.pdf\#page $=57$

21. Periáñez $M$, Ruiz-Drebing $M$, Corbera J.A, Peña $M$, Rodón J, Melián C. Hipertiroidismo felino en España: estudio de prevalencia y de la utilidad diagnóstica de la medición de TSH canina. Clin. Vet. Peq. Anim, 2015, 35 (3): 177 - 182.

22. Bertoy R. Megacolon in the cat. VetClin North Am Small AnimPract. [Internet] 2002 Jul; 32(4) 901-915. Recuperado de: https://europepmc.org/articl e/med/12148317

23. Martín F. Megacolon en gatos: tratamiento con acupuntura. Vol. 1 Num 2 Octubre/ Diciembre 2007. Rev. Inter. de Acupuntura.

24. Todorova I. Prevalence and etiology of the mostcom monmalignant tumours in dogs and cats. Bulg. J. Vet. Med. 2006. 9, No 2, 85-98.

25. Gerber B, Boretti F, Kley S, et al. Evaluation of clinicalsigns and causes of lowerurinar ytract disease in Europeancats. J Small Anim Pract. [Internet] 2005; 46(12): 571-577. Recuperado de: https://pubmed.ncbi.nlm.nih.gov/16355731/

26. Gunn-Moore D. Feline lower urinary tract disease. Journalof FelMed and Sur. 2003. 5(2), 133-138. https://doi.org/10.1016/S1098-612X(02)00129-8

27. Ortiz Álvarez JF. Linfoma linfocítico difuso en el íleon de un felino: informe clínico-patológico. Rev Med Vet. 2015;(30):117-27.

28. Hinostroza E, Grandez R, Salgado S, Salas E. Respuesta a la quimoterapia contra linfoma mediastínico en un gato doméstico. RevlnvVet Perú 2018; 29(4): 1548-1555.

29. Ferasin L, Sturgess C, Cannon M, Caney S, Gruffydd-Jones T, Wotton P. Felineidio pathiccar diomyo pathy: a retrospective study of 106 cats (1994-2001). J Feline Med Surg. [Internet] 2003; 5(3): 151-159. Recuperado de: https://pubmed .ncbi.nlm.nih.gov/12765625/

30. Rush JE, Freeman LM, Fenollosa NK, Brown DJ. Population and survival characteristics of catswith hypertrophic cardiomyopathy: 260 cases (19901999). J Am Vet Med Assoc. 2002; $220(2): 2$ 02-207. Recuperado de: https://pubmed.ncbi.nlm. nih.gov/12126131/ 